\title{
The Importance of Palliative Care in COVID-19
}

\author{
Orkun Saricam $^{1 *}$ (D), Dogan Akdogan ${ }^{2}$ \\ ${ }^{1}$ Department of Internal Medicine Clinic, Pursaklar State Hospital, Ankara, Turkey \\ ${ }^{2}$ Department of Clinic Microbiology, Ankara Pursaklar State Hospital, Ankara, Turkey \\ * Corresponding author: Orkun Saricam E-mail: orkunsar@hotmail.com ORCID: 0000-0001-5820-0951 \\ Received: 17 May 2021 Accepted: 7 September 2021
}

\begin{abstract}
Palliative care, special medical care for a person maintaining his/her life with a severe illness, is a philosophy of care and clinical specialty that improves the life quality of the patient and his/her family, providing relief from the symptoms and stress of life-threatening diseases. For families and healthcare professionals, caring for COVID-19-infected patients require complex symptom management, specific care goals, and psychosocial support. There are studies in the literature showing many favorable aspects of palliative care enabling achievements such as improvements in the quality of life, a shortened length of hospital stay, and reduced costs of care. Palliative care should be provided to all patients in need, regardless of their prognosis, and patients should have access to such life-supporting interventions.
\end{abstract}

Keywords: COVID-19, palliative care, respiratory failure

\section{DEFINITION AND PATHOPHYSIOLOGY}

Coronaviruses are RNA viruses, affecting many body systems in a variety of hosts. The consequences of coronavirus infection can vary from simple upper respiratory tract infection symptoms to the occurrence of severe respiratory diseases such as severe acute respiratory syndrome (SARS) and Middle East respiratory syndrome (MERS) [1]. The SARSCoV-2 virus emerged in China and led to the current pandemic, which has not been under control yet. Although its pathophysiology is complex and not fully understood, it is thought that, like SARS-CoV-1, SARS-CoV-2 primarily targets the lung's epithelial cells and induces the production of pro-inflammatory cytokines and a simultaneous reduction in T-lymphocyte response [2].

\section{DIAGNOSIS AND CLINIC}

COVID-19 spreads quickly. A PCR test is the gold standard in the diagnosis. SARS-CoV-2 has been detected in the respiratory tract on oropharyngeal and nasopharyngeal swabs, in the sputum, and in bronchial fluid samples. The highest viral load is observed in the lower respiratory tract [3].

SARS-CoV-2 can infect individuals from any age group. The most common symptoms of COVID-19 are fever, myalgia, fatigue, dry cough, dyspnea, losses in the senses of taste and smell, and pneumonia. The incubation period is 2 to 14 days [4]. It has been observed that the likelihood of the occurrence of severe respiratory disease and hospital admission is high in persons with chronic diseases and especially in men over the age of 60 years. Children and

() 2021 by the authors; licensee EJMETS by Bastas, UK. This article is an open access article distributed under the terms and conditions of the Creative Commons Attribution License (http://creativecommons.org/licenses/by/4.0/). 
adolescents usually suffer from an asymptomatic or mild disease (non-pneumonia or mild pneumonia) [5]. To date, there is no drug approved for treatment. For families and healthcare professionals, COVID-19-infected patients require complex symptom management, specific care goals, and psychosocial support.

\section{PALLITATIVE CARE}

Palliative care, special medical care for a person maintaining his/her life with a severe illness, is a philosophy of care and clinical specialty that improves the life quality of the patient and his/her family, providing relief from the symptoms and stress of life-threatening diseases [6].Palliative care philosophy states that every person deserves the best quality of life regardless of health conditions and social conditions.

There have been efforts to integrate palliative care with intensive care, emergency services, nursing home services, and home care services for the last 10 years. Applying either aggressive or less aggressive palliative care in emergency room settings based on patients' clinical characteristics has a critical potential to meet the treatment needs of patients during the COVID-19 pandemic. In cases of medical-ethical dilemmas, the integration of palliative care services into patient management is important for developing a patientcentered treatment goal. However, there is a paucity of literature on how physicians should provide palliative care services to COVID-19 patients under emergency room settings [7].

\section{PALLITATIVE CARE APPROACH FOR A COVID-19 PATIENT}

In the COVID-19 pandemic, patients have access problems to hospitals and intensive care units while complying with physical distance and isolation rules. Patients' psychosocial and moral support needs are growing during the pandemic because of the barriers in communication with loved ones and due to the difficulties in the sustainability of palliative care services [8]. Radbruch et al. [9] have emphasized the importance of optimizing cooperation and coordination during the pandemic, maintaining the continuity of care, improving social support, and evaluating emerging needs $A$ multidisciplinary action is needed by taking care of patients and their families, although there are difficulties in the pandemic due to patient isolation, staff restrictions, and limited resources, such as ventilators and hospital beds [10].

Based on risk/benefit evaluations; glucocorticoids, cytokine inhibitors, antiviral agents, and chloroquine/hydroxychloroquine are used in the treatment of COVID-19. In addition to these agents, oxygen therapy is given to patients with severe symptoms. Mechanical ventilation is necessary when patients progress to respiratory failure and become resistant to oxygen therapy. There must be an awareness that respiratory failure may represent a terminal event of severe comorbidity in COVID19 infection. Palliative care is particularly important because it aims to alleviate stressful symptoms in the best way possible [11].

Morphine is the first choice for shortness of breath, cough, and pain. Oxygen can be delivered at 5-10 L/min. without the need for saturation measurement and can be cut if the patient does not recover within an hour. It is important to provide a calm environment. In non-intubated patients with respiratory failure, symptoms of anxiety and panic, as well as shortness of breath, should be closely recorded and treated as soon as possible [12]. Human contact (with adequate protective equipment) should be maintained to prevent feelings of loneliness and vulnerability and improve patients' psychosocial health.

In the treatment of COVID-19, it is necessary to benefit from complementary and alternative therapy methods besides drug therapies after considering the clinical condition of the patient, the duration of infection, and individual differences. The integration of the palliative medicine specialty into such treatment approaches will provide guidance through complex medical-ethical dilemmas that are encountered in the management of patient-centered treatment goals [13].

\section{CONCLUSION}

The COVID-19 pandemic has increased the demand for palliative care services and created both literal walls and barriers between healthcare professionals and families. For patients with life-limiting diseases, palliative care services are complementary to aggressive treatments in acute hospitals, as well as to reduce the symptom burden of patients. There is evidence to demonstrate that palliative care shortens the length of hospital stay, improves the quality of life, and provides better outcomes including acute care outcomes. Regardless of the prognosis, palliative care services should be provided to all patients; who need such care. It should be ensured that patients should have access to life-supporting interventions.

Author contributions: All authors were involved in concept, design, collection of data, interpretation, writing, and critically revising the article. All authors approve final version of the article.

Funding: The authors received no financial support for the research and/or authorship of this article.

Declaration of interest: Authors declare no competing interest. 
Data availability: Data generated or analysed during this study are available from the authors on request.

\section{REFERENCES}

1. Lu R, Zhao X, Li J, et al. Genomic characterization and epidemiology of 2019 novel coronavirus: implications for virus origins and receptor binding. Lancet. 2020.

2. Fehr A, Perlman S. Coronaviruses: an overview of their replication and pathogenesis. Methods Mol Biol. 2015; 1282: 1-23. (doi: 10.1007/978-1-4939-2438-7_1).

3. Konrad R, Eberle U, Dangel A, et al. Rapid establishment of laboratory diagnostics for the novel coronavirus SARSCoV-2 in Bavaria, Germany, February 2020. Euro Surveill. 2020; 25: 2000173. (doi: 10.2807/15607917.ES.2020.25.9.2000173).

4. Huang C, Wang Y, Li X, et al. Clinical features of patients infected with 2019 novel coronavirus in Wuhan, China. Lancet. 2020; 395: 497-506. (doi: 10.1016/S01406736(20)30183-5).

5. Guan WJ, Ni Z, Hu Y, et al. Clinical characteristics of coronavirus disease 2019 in China. N. Engl J Med. 2020; 382: 1708-20. (doi: 10.1056/NEJMoa2002032).

6. Sarıçam G, Kahveci K, Akdoğan D. Palliative care requirement in neurologic diseases. Turk J Neurol. 2020; 26: 153-59. (doi: 10.4274/tnd.2020.26594).

7. Eygnor JK, Rosenau AM, Burmeister DB, et al. Palliative care in the emergency department during a COVID-19 pandemic. Am J Emerg Med 2020 Jul 5. (doi: 10.1016/j.ajem.2020.07.004).
8. Hazini A, Mohammadpour S, Soleimanpour S. The role of palliative care during the coronavirus (COVID-19) pandemic. Int J Palliat Nurs. 2020; 26: 198-99. (doi: 10.12968/ijpn.2020.26.5.198).

9. Radbruch L, Knaul FM, Lima LD, Joncheere CD, Bhadelia A. The key role of palliative care in response to the COVID-19 tsunami of suffering. Lancet. 2020; 395(10235): 1467-69. (doi: 10.1016/S0140-6736(20)30964-8).

10. Feder SL, Akgün KM, Green DS. Palliative care strategies offer guidance to clinicians and comfort for COVID-19 patient and families. Heart Lung. 2020; 49: 227-28. (doi: 10.1016/j.hrtlng.2020.04.001).

11. Pfeifer M, Ewig S, Voshaar T. Positionspapier zur praktischen Umsetzung der apparativen Differenzialtherapie der akuten respiratorischen Insuffizienz bei COVID-19. Pneumologie. 2020; 74(06): 337-57. (doi: 10.1055/a-1157-9976).

12. Ethikrat D. Solidarität und Verantwortung in der CoronaKrise. Ad-hoc-Empfehlung Berlin:2020. Medizinrecht. 2020; 38(6): 466-71. (doi: 10.1007/s00350-020-5563-6).

13. Zhang K, Tian M, Zeng Y, et al. The combined therapy of a traditional Chinese medicine formula and Western medicine for a critically ill case infected with COVID-19. Complement Ther Med. 2020; 52: 102473. (doi: 10.1016/j.ctim.2020.102473). 